\title{
Ri-gene transformation into leaf tissues of Artemisia annua L. and cultivation of hairy-root for artemisinin accumulation
}

\author{
Tran Van Minh
}

\begin{abstract}
The medium B5 was favored for root initiation. Strong initiation of root was leaves used as materials supplemented with NAA $(0.5 \mathrm{mg} / \mathrm{l})$. Cytokinin (BA, kinetin) and IAA, IBA were not favored for root initiation cultures. Leaves of in vitro plantlets were used as materials for gene transformation. The method of agrobacterium for gene transformation was effectiveness. To obtain the leaves having gene transformation and release agrobacterium. Hairy-roots were established on medium of B5 + NAA $(0.5 \mathrm{mg} / \mathrm{l})$ and used as materials for liquid culture research. The modified MS liquid-media having sucrose $(50 \mathrm{~g} / \mathrm{l})$, regulating of $\mathrm{NH}^{+} / \mathrm{NO3}^{-}(5 / 25)$, supplemented with NAA $(0.5 \mathrm{mg} / \mathrm{l})$ enhance artemisinin accumulation up to $1.468 \%$.
\end{abstract}

Keywords-Artemisia annua L., Ri-gene, gene transformation, hairy-root, accumulation

\section{Introduction}

Artemisinin is a potent antimalarials nowadays, but artemisinin biosynthesis is still under study and low levels of naturally occurring exotic plants are extracted from Artemisia Annu L. of $0.01 \%-0.5 \%$ relative to dry weight and high content when flowering is just blossoming at flowering stage [1]. The artemisinin biosynthesis is difficult due to its complex structure, depending on the variety, cultivation conditions and geography. Chlomequat has been used to raise artemisinin levels but there is no effective way to provide adequate artemisinin to the 490 million people who are sick and block millions of deaths annually.

Culture tissue plays an important role in gene transfer. Many transgenic studies on tissue culture of Artemisia Annu L. usually use young culture material. However, there are also many studies using cell suspension. In root culture studies, root-forming and non-callus tissue forms, resulting in high root growth efficiency in liquid culture on a shaker or bioreactor [2].

\section{Tran Van Minh,}

International University,

Vietnam National University Ho Chi Minh City, Vietnam

Linh Trung Ward, Thu Duc District, Ho Chi Minh City

Vietnam

Tel: $84-28-32744720$

Fax: 84-28-32744721
In order to obtain high yielding root crops, improved strains begin with the choice of parent plants that the desired product is high in. The different strains of agrobaterium rhizogenes may also affect gene transfer. Giri \& Narasu [3] showed the contrast of A4, 15834, K599, LBA 9402, and 9340 strains of A. Rhizogenes, of which LBA 9365 was found to induce A. Annua with higher artemisinin content. However, some other plant species may respond better to other strains of A. Rhizogenes. Successful gene transfer to callus tissue regeneration shoots [4], suspension [5] and roots [6, 7, 8] have resulted in increased levels of artemisinin synthesized in the cell 1-5 times.

As hair- root of Artemisia Annu L. can accumulate artemisinin, there are many studies on nutrient environment, growth regulator, protein, sugar and light that affect artemisinin synthesis through haity-root culture on liquid medium $[2,9,10,11,12,13]$. In this paper, we study the transformation of hairy-root gene into leaf tissue and influence of liquid culture medium on accumulation of artemisinin content.

\section{Material and Methods}

\section{A. Selecting Transgenic Root Tissue \\ 1) Material}

Artemisia Annu L. bean TC1.8 selected lines were sown on MS medium in vitro. Tissue culture is used as a culture material. Leaves, stems and callus tissue are used for root growth.

\section{2) The culture medium}

B5 [1], MS [8], LV [6] supplemented with: Kinetin (furfuryl aminopurine), BA (benzylaminopurine), IAA ( $\beta$ indoleacetic acid), IBA ( $\beta$-indolebutyric acid), NAA $(\alpha-$ naphthaleneacetic acid), 2,4D (di-chlorophenoxyacetic acid).

\section{3) Conditions of culture}

Temperature $28+2^{\circ} \mathrm{C}, \mathrm{RH}=65 \%$, Light intensity 22.2 $\mathrm{mmol} / \mathrm{m}^{2} / \mathrm{s}$, lighting time 10 hours/day.

\section{4) Method}

The experiment was arranged randomly, three times, three times with 3 triangles, each with 7 leaves. Data were recorded after 30 days of culture and analyzed by MSTATC statistical program $(\mathrm{p}=0.05)$. 
Proc. of The Seventh Intl. Conf. On Advances in Applied Science and Environmental Technology - ASET 2017 Copyright (C) Institute of Research Engineers and Doctors, USA .All rights reserved.

ISBN: 978-1-63248-136-8 doi: 10.15224/ 978-1-63248-136-8-26

\section{5) Experimental design}

Root formation medium: Use three mineral media MS, B5, LV supplemented with growth regulators of auxin and cytokinin: On semi-solid medium B5 supplemented with BA, Kinetin, IAA, IBA, NAA or $2,4 \mathrm{D}$ concentrations $(0,0.1,0.5$, $0.7,1 \mathrm{mg} / \mathrm{l})$.

Effect of culture tissue: Explants culture (stem $10 \mathrm{~mm}$ ), leaves $(1 \times 1 \mathrm{~cm})$, callus tissue. The semi-solid medium for root formation cultured with B5 supplemented with IAA, IBA and NAA concentrations of $0.1-0.5 \mathrm{mg} / \mathrm{l}$.

\section{B. Transferring the Hairy-Root Gene into Leaf Tissue of Artemisia Annu L.}

\section{1) Material}

Agrobaterium ATCC 11325 imported from the United States (ITS company). The seedlings of line TC1.8 were obtained from the HCMC selection experiment. TC1 grains were supplied by Hanoi research center for medicinal materials.

Agrobacterium strain cultured on LB medium (Luria Bertani): Tryton $10 \mathrm{~g} / \mathrm{l}, 5 \mathrm{~g} / \mathrm{l}$ yeast extract, $\mathrm{NaCl} 10 \mathrm{~g} / \mathrm{l}, \mathrm{PH}$ 7.2. To propagate the gene transfer, the bacteria were shaken overnight in an LB medium at $26^{\circ} \mathrm{C}$.

Sow seeds in vitro was that the experiment was conducted in a process that included: Seeds were washed through the savon for 3-5 minutes. Wear the washed bead on the sterile incubator. Wash with sterile distilled water 2-3 times. Shake in $70 \%$ ethanol for 1 minute, rinse with sterile distilled water 3 to 5 times. Soak in hypochlorite solution for 30 minutes. Rinse the sterile solution with sterile distilled water 3-5 times. Place sterilized seeds in $250 \mathrm{ml}$ Erlene containing $50 \mathrm{ml}$ of base MS medium. One month after seed germination will be transferred to MS medium without plant growth regulator.

Genetic transformation process: The fact that artemisinin is synthesized and stored almost exclusively in leaves of Artemisia Апnи L. , the leaves are used as a source of material for hairy-root gene transfer to produce artemisinin. The mature leaves from in vitro seedlings were cut to $0.3 \times 0.3 \mathrm{~cm}$ for wound formation. Inoculation of leaf tissue with bacteria for 30 minutes. Gradually remove the excess bacteria from the filter paper. Culture of leaf pieces with bacteria in B5 supplemented with acetosyringone (AS) $100 \mu \mathrm{m}$ for 2 days. Rinse the bacteria with sterile distilled water. Dissolve the sample, drain and cultured the leaf pieces on B5 medium supplemented with $500 \mathrm{mg} / \mathrm{l}$ Cefotaxime to kill the remaining bacteria. Culturing time of 3 weeks was changed to new medium. Repeat 3 times. Observe root formation from transformed leaf tissue samples. Evaluation of the ability to remove Agrobacterium by dry root powder ELISA.

\section{2) Design experiment}

Establishment of roots in transgenic leaves: Transgenic plants were cultured on B5, MS, LV regenerated roots supplemented with NAA growth regulators $(0,0.1,0.5,0.7,1$ $\mathrm{mg} / \mathrm{l})$. Tracking indicator is the number of roots formed on cultured specimens after 30 culture days.

Growth of transgenic roots: The roots of 10-30 mm length were cultured in B5 + NAA medium $(0.5 \mathrm{mg} / \mathrm{l})$.

\section{Culture of Transgenic Hairy-Root on Liquid Medium \\ 1) Material}

Artemisia Anпu L. grain line TC1.8 line selected

MS, B5, LV medium supplemented with NAA (0.5 mg /l), BA, GA3 (Gibberellic Acid)

\section{2) Conditions of culture}

Temperature $28+2{ }^{\circ} \mathrm{C}, \mathrm{RH}=65 \%$, light intensity 22.2 $\mu \mathrm{mol} / \mathrm{m}^{2} / \mathrm{s}$, lighting time 10 hours/day.

\section{3) Method}

The experiment was arranged randomly, three times each, three times with flasks (50 ml culture medium), each with a flask implanted with $100 \mathrm{mg}$ root sample. Data were collected after 21 culture days and analyzed by MSTATC statistical program $(\mathrm{p}=0.05)$.

\section{4) Design experiment}

a) Effects of nutrient medium on artemisinin accumulation

Hairy-root was cultured in two B5 and MS mediums, supplemented with NAA $(0.5 \mathrm{mg} / \mathrm{l})$.

\section{b) Effect of sucrose on artemisinin} accumulation

Hairy-root was cultured on MS + NAA medium $(0.5 \mathrm{mg} / \mathrm{l})$ supplemented with sucrose $(0,10,20,30,40,50,60,70,80$, $90 \mathrm{~g} / \mathrm{l})$.

\section{c) Effect of nitrate nitrogen on} artemisinin accumulation

The hairy-root cultured on MS + NAA $(0.5 \mathrm{mg} / \mathrm{l})+$ sucrose $(50 \mathrm{~g} / \mathrm{l})$ supplemented with nitrate nitrogen $(10,20$, $30,40,50,60,70,80,90 \mathrm{mM})$.

\section{d) Effect of $\mathrm{NH}^{+} / \mathrm{NO}^{-}$on artemisinin accumulation}

The hairy-root grown on MS medium + NAA $(0.5 \mathrm{mg} / \mathrm{l})+$ sucrose $(50 \mathrm{~g} / \mathrm{l}) \mathrm{NH}^{+}{ }^{+}$NO3 $^{-}(0 / 30,5 / 25,10 / 20,15 / 15,20 / 10$, $25 / 5,30 / 0)$.

\section{e) Effect of inorganic phosphate on artemisinin accumulation}


Proc. of The Seventh Intl. Conf. On Advances in Applied Science and Environmental Technology - ASET 2017 Copyright (C) Institute of Research Engineers and Doctors, USA .All rights reserved.

ISBN: 978-1-63248-136-8 doi: 10.15224/ 978-1-63248-136-8-26

The hairy-root was cultured on MS + NAA medium $(0.5$ $\mathrm{mg} / \mathrm{l})+$ sucrose $(50 \mathrm{~g} / \mathrm{l})$ supplemented with $\mathrm{KH}_{2} \mathrm{PO}_{4}(0.5-1,0$ $1,5-2,0-2,5-3,0-3,5 \mathrm{~mm})$.

\section{f) Effect of BA on artemisinin accumulation}

The hairy-root cultured on MS + NAA medium $(0.5 \mathrm{mg} / \mathrm{l})$ + sucrose $(50 \mathrm{~g} / \mathrm{l})$ supplemented with BA $(0-0,1-0,3-0,5-0$, 7-1, $0 \mathrm{mg} / \mathrm{l})$.

\section{g) Effect of GA3 on artemisinin accumulation}

The hairy-root was cultured in MS + NAA medium (0.5 $\mathrm{mg} / \mathrm{l})+$ sucrose $(50 \mathrm{~g} / \mathrm{l}$ supplemented with GA3 $(0,1,2,3,4$, $5,6,7 \mathrm{mg} / \mathrm{l})$.

\section{h) Modified medium}

The hairy-root was cultured in MS and modified MS medium supplemented with NAA $(0.5 \mathrm{mg} / \mathrm{l}), \mathrm{NH}^{+} / \mathrm{NO}^{-}$ $(5 / 25)$, sucrose $50 \mathrm{~g} / \mathrm{l}$.

\section{g) Culture of Artemisia Annu L. hairy- root on Temporary Immersion Bioreactor}

Growth dynamics of the roots on temporary immersion system bioreactor: The hairy-root was cultured in a modified MS medium. Time for growth dynamics (0, 5, 10, 15, 20, 25 days after culture) at temporary at floating 1 minutes and sinking 4 hours.

\section{Results and Discussion}

\section{A. Selecting Tissue Culture for Transgenic Hairy-Root}

\section{1) Root formation medium}

There are currently three major base medium in labs in the world that are used extensively in rootstock research, MS, B5 and LV. The goal was to choose the right nutrient medium for root regeneration and without the formation of callus tissue in leaves or in newly formed hairy-root. The results showed that B5 medium was rooted and did not form callus tissue from culture leave tissue (Table I).
TABLE I. EFFECT OF BASE MEDIUM ON ROOT REGENERATION

\begin{tabular}{|c|c|c|c|c|c|c|}
\hline Base & \multicolumn{2}{|c|}{ IAA (mg/l) } & \multicolumn{2}{c|}{ IBA (mg/l) } & \multicolumn{2}{c|}{ NAA (mg/l) } \\
\cline { 2 - 7 } medium & $\mathbf{0 . 1}$ & $\mathbf{0 . 5}$ & $\mathbf{0 . 1}$ & $\mathbf{0 . 5}$ & $\mathbf{0 . 1}$ & $\mathbf{0 . 5}$ \\
\hline MS & Creates & Creates & Creates & Creates & Creates & Creates \\
& more & more & more & more & more & more \\
& roots, & roots, & roots, & roots, & roots, & roots, \\
& less & less & less & less & less & less \\
& callus & callus & callus & callus & callus & callus \\
& tissue & tissue & tissue & tissue & tissue & tissue \\
\hline B5 & Create & Create & Create & Create & Create & Create \\
& many & many & many & many & many & many \\
& roots & roots & roots & roots & roots & roots \\
\hline LV & Create & Create & Create & Create & Create & Create \\
& many & many & many & many & many & many \\
& roots, & roots, & roots, & roots, & roots, & roots, \\
& many & many & many & many & many & many \\
& callus & callus & callus & callus & callus & callus \\
& tissue & tissue & tissue & tissue & tissue & tissue \\
\hline
\end{tabular}

2) Growth regulators auxin and cytokinin

Cytokinin (BA, Kinetin) was not affect root regeneration, yellowing and non-callus tissue formation. Auxins (IAA, IBA, NAA, 2,4D) play an important role in the formation of roots, which arise in most concentrations. The control was not develop root formation, after 7 days the tissues turn yellowish. Of which NAA $(0.5 \mathrm{mg} / \mathrm{l})$ gave more root regeneration than the other auxin (Table II).

TABLE II. EFFECT OF CYTOKININ AND AUXIN ON THE ABILITY OF CULTURED CALLUS TISSUE REGENERATION

\begin{tabular}{|c|c|c|c|c|c|}
\hline $\begin{array}{c}\text { Concentration } \\
(\mathrm{mg} / \mathrm{l})\end{array}$ & 0 & 0.1 & 0.5 & 0.7 & 1 \\
\hline $\mathrm{BA}$ & $\begin{array}{c}\text { Not } \\
\text { responding }\end{array}$ & $\begin{array}{l}\text { Yellow, } \\
\text { wilt, } \\
\text { not } \\
\text { create } \\
\text { callus } \\
\text { tissue }\end{array}$ & $\begin{array}{c}\text { Yellow, } \\
\text { wilt, } \\
\text { not } \\
\text { create } \\
\text { callus } \\
\text { tissue }\end{array}$ & $\begin{array}{l}\text { Yellow, } \\
\text { wilt, } \\
\text { not } \\
\text { create } \\
\text { callus } \\
\text { tissue }\end{array}$ & $\begin{array}{c}\text { Yellow, } \\
\text { wilt, } \\
\text { not } \\
\text { create } \\
\text { callus } \\
\text { tissue }\end{array}$ \\
\hline Kinetin & $\begin{array}{c}\text { Not } \\
\text { responding }\end{array}$ & $\begin{array}{c}\text { Yellow, } \\
\text { wilt, } \\
\text { not } \\
\text { create } \\
\text { callus } \\
\text { tissue } \\
\end{array}$ & $\begin{array}{c}\text { Yellow, } \\
\text { wilt, } \\
\text { not } \\
\text { create } \\
\text { callus } \\
\text { tissue } \\
\end{array}$ & $\begin{array}{c}\text { Yellow, } \\
\text { wilt, } \\
\text { not } \\
\text { create } \\
\text { callus } \\
\text { tissue }\end{array}$ & $\begin{array}{c}\text { Yellow, } \\
\text { wilt, } \\
\text { not } \\
\text { create } \\
\text { callus } \\
\text { tissue } \\
\end{array}$ \\
\hline IAA & $\begin{array}{c}\text { Not } \\
\text { responding }\end{array}$ & $\begin{array}{c}\text { Create } \\
\text { many } \\
\text { roots }\end{array}$ & $\begin{array}{c}\text { Create } \\
\text { many } \\
\text { roots }\end{array}$ & $\begin{array}{c}\text { Create } \\
\text { many } \\
\text { roots }\end{array}$ & $\begin{array}{c}\text { Create } \\
\text { many } \\
\text { roots }\end{array}$ \\
\hline IBA & $\begin{array}{c}\text { Not } \\
\text { responding }\end{array}$ & $\begin{array}{c}\text { Create } \\
\text { many } \\
\text { roots }\end{array}$ & $\begin{array}{c}\text { Create } \\
\text { many } \\
\text { roots }\end{array}$ & $\begin{array}{c}\text { Create } \\
\text { many } \\
\text { roots }\end{array}$ & $\begin{array}{c}\text { Create } \\
\text { many } \\
\text { roots }\end{array}$ \\
\hline NAA & $\begin{array}{c}\text { Not } \\
\text { responding }\end{array}$ & $\begin{array}{c}\text { Create } \\
\text { many } \\
\text { roots }\end{array}$ & $\begin{array}{c}\text { Create } \\
\text { many } \\
\text { roots }\end{array}$ & $\begin{array}{c}\text { Create } \\
\text { many } \\
\text { roots }\end{array}$ & $\begin{array}{c}\text { Create } \\
\text { many } \\
\text { roots }\end{array}$ \\
\hline $2,4 \mathrm{D}$ & $\begin{array}{c}\text { Not } \\
\text { responding }\end{array}$ & $\begin{array}{c}\text { Create } \\
\text { many } \\
\text { roots }\end{array}$ & $\begin{array}{c}\text { Create } \\
\text { many } \\
\text { roots }\end{array}$ & $\begin{array}{l}\text { Create } \\
\text { many } \\
\text { roots }\end{array}$ & $\begin{array}{c}\text { Create } \\
\text { many } \\
\text { roots }\end{array}$ \\
\hline
\end{tabular}

3) Effect of culture tissue

Callus and root formation from leave in auxin and culture concentration, strong root formation in leaves with NAA concentrations $(0.5 \mathrm{mg} / \mathrm{l})$ (Table III). 
Proc. of The Seventh Intl. Conf. On Advances in Applied Science and Environmental Technology - ASET 2017 Copyright (C) Institute of Research Engineers and Doctors, USA .All rights reserved.

ISBN: 978-1-63248-136-8 doi: 10.15224/ 978-1-63248-136-8-26

TABLE III. EFFECT OF TISSUE CULTURE ON ROOT REGENERATION

\begin{tabular}{|c|c|c|c|c|c|c|}
\hline \multirow{2}{*}{$\begin{array}{c}\text { Base } \\
\text { medium }\end{array}$} & \multicolumn{2}{|c|}{ IAA $(\mathrm{mg} / \mathrm{l})$} & \multicolumn{2}{|c|}{ IBA (mg/l) } & \multicolumn{2}{|c|}{ NAA (mg/l) } \\
\hline & 0.1 & 0.5 & 0.1 & 0.5 & 0.1 & 0.5 \\
\hline $\begin{array}{c}\text { Leave } \\
(0,3 \mathrm{x} \\
0,3 \mathrm{~cm})\end{array}$ & $\begin{array}{l}\text { Create } \\
\text { many } \\
\text { roots }\end{array}$ & $\begin{array}{l}\text { Create } \\
\text { many } \\
\text { roots }\end{array}$ & $\begin{array}{l}\text { Create } \\
\text { many } \\
\text { roots }\end{array}$ & $\begin{array}{l}\text { Create } \\
\text { many } \\
\text { roots }\end{array}$ & $\begin{array}{c}\text { Create } \\
\text { many } \\
\text { roots }\end{array}$ & $\begin{array}{c}\text { Create } \\
\text { many } \\
\text { roots }\end{array}$ \\
\hline $\begin{array}{c}\text { Stem } \\
(10 \mathrm{~mm})\end{array}$ & $\begin{array}{c}\text { Create } \\
\text { roots at } \\
\text { two } \\
\text { cuts, } \\
\text { less } \\
\text { roots }\end{array}$ & $\begin{array}{c}\text { Create } \\
\text { roots at } \\
\text { two } \\
\text { cuts }\end{array}$ & $\begin{array}{l}\text { less } \\
\text { roots }\end{array}$ & $\begin{array}{c}\text { Create } \\
\text { roots at } \\
\text { two } \\
\text { cuts }\end{array}$ & $\begin{array}{l}\text { less } \\
\text { roots }\end{array}$ & $\begin{array}{c}\text { Create } \\
\text { roots at } \\
\text { two } \\
\text { cuts }\end{array}$ \\
\hline $\begin{array}{c}\text { Callus } \\
\text { (Ф5 } \\
\text { mm) }\end{array}$ & $\begin{array}{l}\text { Create } \\
\text { medium } \\
\text { roots }\end{array}$ & $\begin{array}{l}\text { Create } \\
\text { medium } \\
\text { roots }\end{array}$ & $\begin{array}{l}\text { Create } \\
\text { medium } \\
\text { roots }\end{array}$ & $\begin{array}{c}\text { Create } \\
\text { medium } \\
\text { roots }\end{array}$ & $\begin{array}{c}\text { Create } \\
\text { medium } \\
\text { roots }\end{array}$ & $\begin{array}{c}\text { Create } \\
\text { medium } \\
\text { roots }\end{array}$ \\
\hline
\end{tabular}

B. Transferring the Hairy-Root Gene into Leaf Tissue of Artemisia Annu L. leaves

1) Regeneration of roots in transgenic

All three MS, B5, LV media were suitable for root regeneration. B5 + NAA $(0.5 \mathrm{mg} / \mathrm{l})$ was used in $\mathrm{B} 5+$ medium (10.2 roots/culture sample) and did not form callus tissue. On MS and LV media, root regeneration was associated with callus tissue formation (Table IV).

TABLE IV. REGENERATING ROOTS (NUMBER OF ROOTS/CULTURE SAMPLE) IN VITRO

\begin{tabular}{|l|l|l|l|}
\hline \multicolumn{1}{|c|}{ NAA } & \multicolumn{1}{c|}{ MS $(*)$} & \multicolumn{1}{c|}{ B5 } & \multicolumn{1}{c|}{$\mathbf{L V}(*)$} \\
\hline 0.0 & 3.2 & 4.6 & 1.4 \\
\hline 0.1 & 4.2 & 5.8 & 2.8 \\
\hline 0.5 & 9.6 & 10.2 & 6.8 \\
\hline 0.7 & 6.2 & 9.8 & 4.2 \\
\hline 1.0 & 4.5 & 8.2 & 3.4 \\
\hline $\mathrm{CV}(\%)$ & 14.2 & 12.6 & 16.4 \\
\hline
\end{tabular}

(*): Create roots and callus tissue

\section{2) Growth of transgenic roots}

On B5 + NAA medium $(0.5 \mathrm{mg} / \mathrm{l})$, the root growth unit (RGU) reached the highest (3.25) on day 14 and subsequently decreased due to the development of the number of hairy-root (7.4) faster growth of main root length $(9.8 \mathrm{~cm})$ at day 28 (Table V).

TABLE V. GROWTH OF HAIRY-ROOT

\begin{tabular}{|l|l|l|l|l|}
\hline \multicolumn{1}{|c|}{ Day after culture } & \multicolumn{1}{|c|}{$\mathbf{7}$} & $\mathbf{1 4}$ & $\mathbf{2 1}$ & $\mathbf{2 8}$ \\
\hline Main root length $(\mathrm{cm})$ & 1.20 & 4.50 & 7.60 & 9.80 \\
\hline $\begin{array}{l}\text { Number of hairy-roots } \\
\text { (number of roots) }\end{array}$ & 1.10 & 3.20 & 5.50 & 7.40 \\
\hline $\begin{array}{l}\text { Number of hairy-roots } \\
\text { per cm }\end{array}$ & $0, .91$ & 0.71 & 0.72 & 0.75 \\
\hline Hairy-roots length (cm) & 0.12 & 0.44 & 0.75 & 0.92 \\
\hline $\begin{array}{l}\text { Total hairy-roots length } \\
\text { (cm) }\end{array}$ & 1.30 & 5.90 & 4.10 & 6.80 \\
\hline $\begin{array}{l}\text { RGU root growth unit } \\
(\mathrm{cm})\end{array}$ & 2.74 & 3.25 & 2.12 & 2.24 \\
\hline
\end{tabular}

$R G U($ Root Growth Unit $)=[\Sigma$ a root length of hairy-root + main root length $] /$ number of hairy-roots (McCoy, 2003)

\section{Grow Hairy-Roots of Artemisia Annua L. on Liquid Medium \\ 1) Effects of nutrient medium on artemisinin accumulation}

Fresh root weight was not different in two basic MS and B5 medium; and there was no callus tissue formation. Root culture on semi-solid medium, MS medium with callus tissue. The MS + NAA medium $(0.5 \mathrm{mg} / \mathrm{l})$ was used for liquid culture studies with a concentration accumulation of $1.225 \%$ (Table IV) (Figure 1).

TABLE VI. EFFECT OF NUTRIENT MEDIUM ON THE ACCUMULATION OF ARTEMISININ

\begin{tabular}{|l|l|l|}
\hline \multicolumn{1}{|c|}{ Base medium } & \multicolumn{1}{|c|}{$\begin{array}{c}\text { Dry weight } \\
\text { of roots (g/l) }\end{array}$} & $\begin{array}{c}\text { Art content (\%) } \\
\text { of root dry weight }\end{array}$ \\
\hline B5 & $24.5 \mathrm{ab}$ & 1.146 \\
\hline $\mathrm{MS}$ & $26.2 \mathrm{a}$ & 1.225 \\
\hline $\mathrm{LV}$ & $22.6 \mathrm{~b}$ & 1.086 \\
\hline $\mathrm{CV}(\%)$ & 12.6 & \\
\hline
\end{tabular}

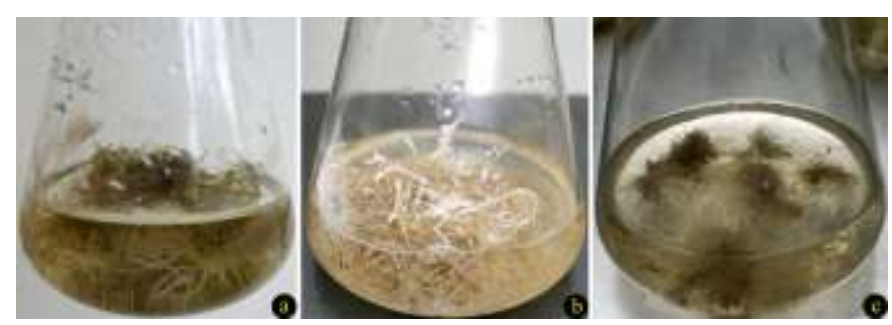

Figure 1. Root growth in the medium of (a) B5 (b) MS (c) LV

\section{2) Effects of sucrose on artemisinin accumulation}

Sugar concentration increased by $10-50 \mathrm{~g} / \mathrm{l}$, artemisinin accumulation increased by $0.750-1.178 \%$; sugar concentration increased 50-90 g/l, the accumulation capacity increased not significantly. Sucrose concentrations increased by more than $70 \mathrm{~g} / \mathrm{l}$, with the possibility of accumulation decreasing. The appropriate culture medium for MS + NAA $(0.5 \mathrm{mg} / \mathrm{l})+$ sucrose $(50 \mathrm{~g} / \mathrm{l})$ had an accumulation of artemisinin content of $1.178 \%$ (Table VII).

TABLE VII. EFFECTS OF SUCROSE ON ARTEMISININ ACCUMULATION

\begin{tabular}{|l|l|l|}
\hline $\begin{array}{c}\text { Sucrose } \\
(\mathbf{g} / \mathbf{l})\end{array}$ & $\begin{array}{c}\text { Dry weight } \\
\text { of roots }(\mathbf{g} / \mathbf{l})\end{array}$ & $\begin{array}{c}\text { Art content } \mathbf{( \% )} \\
\text { of root dry weight }\end{array}$ \\
\hline 10 & 4.5 & 0.750 \\
\hline 20 & 6.8 & 0.902 \\
\hline 30 & 12.1 & 1.089 \\
\hline 40 & 14.5 & 1.146 \\
\hline 50 & 19.2 & 1.178 \\
\hline 60 & 22.5 & 1.182 \\
\hline 70 & 26.2 & 1.196 \\
\hline 80 & 22.8 & 1.202 \\
\hline 90 & 20.5 & 1.212 \\
\hline $\mathrm{CV}(\%)$ & 18.4 & \\
\hline
\end{tabular}


Proc. of The Seventh Intl. Conf. On Advances in Applied Science and Environmental Technology - ASET 2017 Copyright (C) Institute of Research Engineers and Doctors, USA .All rights reserved.

ISBN: 978-1-63248-136-8 doi: 10.15224/ 978-1-63248-136-8-26

\section{3) Effects of nitrate nitrogen on artemisinin accumulation}

Appropriate nitrate concentrations of 30-60 mm, reaching a maximum of $30 \mathrm{~mm}$ for $1.198 \%$ artemisinin accumulation (Table VIII), have not improved significantly artemisinin accumulation

TABLE VIII. EFFECT OF NITRATE NITROGEN ON ARTEMISININ ACCUMULATION

\begin{tabular}{|l|l|l|}
\hline $\begin{array}{c}\text { Nitrate } \\
\text { nitrogen (mM) }\end{array}$ & $\begin{array}{c}\text { Dry weight } \\
\text { of roots (g/l) }\end{array}$ & $\begin{array}{c}\text { Art content (\%) } \\
\text { of root dry weight }\end{array}$ \\
\hline 10 & 15.5 & 0.850 \\
\hline 20 & 22.1 & 0.860 \\
\hline 30 & 25.5 & 1.198 \\
\hline 40 & 26.2 & 1.188 \\
\hline 50 & 25.1 & 1.180 \\
\hline 60 & 25.2 & 1.150 \\
\hline 70 & 20.5 & 1.040 \\
\hline 80 & 17.5 & 0.780 \\
\hline 90 & 13.2 & 0.650 \\
\hline $\mathrm{CV}(\%)$ & 16.2 & \multicolumn{1}{|l|}{} \\
\hline
\end{tabular}

4) Effect of $\mathrm{NH}^{+}+\mathrm{NO3}^{-}$on artemisinin accumulation

Appropriate $\mathrm{NH}^{+}{ }^{+} / \mathrm{NO}^{-}$ratio of $5 / 25$ for $1.386 \%$ accumulation; This was higher than artemisinin concentration (Table IX). Significant improvement of artemisinin accumulation compared with nitrogen fixation $(30 \mathrm{~mm})$.

TABLE IX. EFFECT OF NH4 +/NO3- ON ARTEMISININ ACCUMULATION

\begin{tabular}{|l|l|l|}
\hline \multicolumn{1}{|c|}{$\mathbf{N H 4}^{+} \mathbf{N O 3}^{-}$} & $\begin{array}{c}\text { Dry weight } \\
\text { of roots (g/l) }\end{array}$ & $\begin{array}{c}\text { Art content (\%) } \\
\text { of root dry weight }\end{array}$ \\
\hline $0 / 30$ & 27.2 & 1.315 \\
\hline $5 / 25$ & 28.4 & 1.386 \\
\hline $10 / 20$ & 25.4 & 1.246 \\
\hline $15 / 15$ & 16.8 & 0.984 \\
\hline $20 / 10$ & 10.5 & 0.910 \\
\hline $25 / 5$ & 6.6 & 0.816 \\
\hline $30 / 0$ & 1.5 & 0.714 \\
\hline $\mathrm{CV}(\%)$ & 12.6 & \\
\hline
\end{tabular}

\section{5) Effect of inorganic phosphate on artemisinin accumulation}

Appropriate inorganic phosphate concentration of $1.5 \mathrm{~mm}$ with artemisinin accumulation of $1.316 \%$; inorganic phosphate concentrations were higher, and artemisinin depositional capacity decreases (Table $\mathrm{X}$ ). Inorganic phosphate has not significantly improved the accumulation of artemisinin compared to $\mathrm{NH} 4^{+} / \mathrm{NO}^{-}(5 / 25)$.
TABLE X: EFFECTS OF PHOSPHATE ON ARTEMISININ ACCUMULATION

\begin{tabular}{|l|c|c|}
\hline $\begin{array}{c}\mathbf{K H}_{2} \mathbf{P O}_{4} \\
(\mathbf{m M})\end{array}$ & $\begin{array}{c}\text { Dry weight } \\
\text { of roots (g/l) }\end{array}$ & $\begin{array}{c}\text { Art content (\%) } \\
\text { of root dry weight }\end{array}$ \\
\hline 0.5 & 21.5 & 1.288 \\
\hline 1.0 & 25.0 & 1.298 \\
\hline 1.5 & 23.2 & 1.316 \\
\hline 2.0 & 30.2 & 1.052 \\
\hline 2.5 & 30.4 & 0.754 \\
\hline 3.0 & 30.8 & 0.746 \\
\hline 3.5 & 31.5 & 0.720 \\
\hline $\mathrm{CV}(\%)$ & 14.2 & \\
\hline
\end{tabular}

6) Effect of BA on artemisinin accumulation

Appropriate BA concentration was $0.5 \mathrm{mg} / \mathrm{l}$ for artemisinin accumulation of 1.364\%; higher BA levels, decreased artemisinin accumulation (Table XI). Addition of BA $(0.5$ $\mathrm{mg} / \mathrm{l})$ was not significantly different from that of $\mathrm{NH}^{+}{ }^{+} \mathrm{NO}^{-}$ $(5 / 25)$.

TABLE XI. EFFECT OF BA ON ARTEMISININ ACCUMULATION

\begin{tabular}{|l|l|l|}
\hline \multicolumn{1}{|c|}{ BA (mg/l) } & $\begin{array}{c}\text { Dry weight } \\
\text { of roots (g/l) }\end{array}$ & $\begin{array}{c}\text { Art content (\%) } \\
\text { of root dry weight }\end{array}$ \\
\hline 0.0 & 27.5 & 1.296 \\
\hline 0.1 & 23.4 & 1.312 \\
\hline 0.3 & 23.0 & 1.324 \\
\hline 0.5 & 22.5 & 1.364 \\
\hline 0.7 & 20.2 & 1.278 \\
\hline 1.0 & 18.5 & 1.252 \\
\hline $\mathrm{CV}(\%)$ & 14.2 & \\
\hline
\end{tabular}

\section{7) Effect of GA3 on artemisinin accumulation}

The appropriate GA3 concentration was $5 \mathrm{mg} / \mathrm{l}$ with the highest artemisinin content of $1.303 \%$; higher concentrations of GA3, decreased artemisinin accumulation (Table XII). The GA3 supplementation $(5 \mathrm{mg} / \mathrm{l})$ was not significantly different from the $\mathrm{NH}_{4}{ }^{+} / \mathrm{NO}^{-}(5 / 25)$.

TABLE XII. EFFECT OF GA3 ON ARTEMISININ ACCUMULATION

\begin{tabular}{|l|l|l|}
\hline $\begin{array}{c}\text { BA } \\
(\mathbf{m g} / \mathbf{l})\end{array}$ & $\begin{array}{c}\text { Dry weight } \\
\text { of roots }(\mathbf{g} / \mathbf{l})\end{array}$ & $\begin{array}{c}\text { Art content (\%) } \\
\text { of root dry weight }\end{array}$ \\
\hline 0 & 27.8 & 1.233 \\
\hline 1 & 29.5 & 1.240 \\
\hline 2 & 28.6 & 1.258 \\
\hline 3 & 28.5 & 1.268 \\
\hline 4 & 28.0 & 1.286 \\
\hline $\mathbf{5}$ & $\mathbf{2 7 . 8}$ & $\mathbf{1 . 3 0 3}$ \\
\hline 6 & 27.0 & 1.288 \\
\hline 7 & 26.8 & 1.264 \\
\hline $\mathrm{CV}(\%)$ & 14.2 & \\
\hline
\end{tabular}

\section{8) Modified medium}

On MS medium supplemented with NAA (0.5 mg/l), sucrose $(50 \mathrm{~g} / \mathrm{l})$, $\mathrm{NH} 4{ }^{+} / \mathrm{NO}^{-}(5 / 25)$ improvement of artemisinin accumulation. Nitrate, inorganic phosphate, BA, and GA3 have not contributed to the improvement of artemisinin accumulation. The modified MS medium 
Proc. of The Seventh Intl. Conf. On Advances in Applied Science and Environmental Technology - ASET 2017 Copyright (@) Institute of Research Engineers and Doctors, USA .All rights reserved.

ISBN: 978-1-63248-136-8 doi: 10.15224/ 978-1-63248-136-8-26

improved the biomass growth $(32.6 \mathrm{~g} / \mathrm{l})$ and artemisinin accumulation (1.468\%) (Table XIII).

\section{TABLE XIII. EFFECT OF MODIFIED MS MEDIUM ON ARTEMISININ} ACCUMULATION

\begin{tabular}{|l|l|l|}
\hline Basic medium & \multicolumn{1}{|c|}{$\begin{array}{c}\text { Dry weight } \\
\text { of roots (g/l) }\end{array}$} & $\begin{array}{c}\text { Art content (\%) } \\
\text { of root dry weight }\end{array}$ \\
\hline $\begin{array}{l}\text { MS (not growth } \\
\text { regulator) }\end{array}$ & 28.5 & 1.146 \\
\hline Modified MS & 32.6 & 1.468 \\
\hline CV (\%) & 10.8 & \\
\hline
\end{tabular}

9) Culture of Artemisia annua L. hairyroot in temporary immersion bioreactor

The results of Table XIV show that, on day 20 after culture, artemisinin content reached the highest concentration of $1.775 \%$. In temporary immersion bioreactor, the culture time may be extended to reach the highest root biomass (Figure 2)

TABLE XIV. THE GROWTH DYNAMICS OF HAIR-ROOTS IN TEMPORARY IMMERSION BIOREACTOR

\begin{tabular}{|l|l|l|}
\hline $\begin{array}{c}\text { Time (day } \\
\text { after culture) }\end{array}$ & $\begin{array}{c}\text { Dry weight } \\
\text { of roots (g/l) }\end{array}$ & $\begin{array}{c}\text { Art content (\%) } \\
\text { of root dry weight }\end{array}$ \\
\hline 0 & 0.0 & 1.025 \\
\hline 5 & 4.5 & 0.850 \\
\hline 10 & 12.5 & 1.186 \\
\hline 15 & 19.5 & 1.568 \\
\hline 20 & 26.5 & 1.775 \\
\hline 25 & 27.5 & 1.764 \\
\hline
\end{tabular}

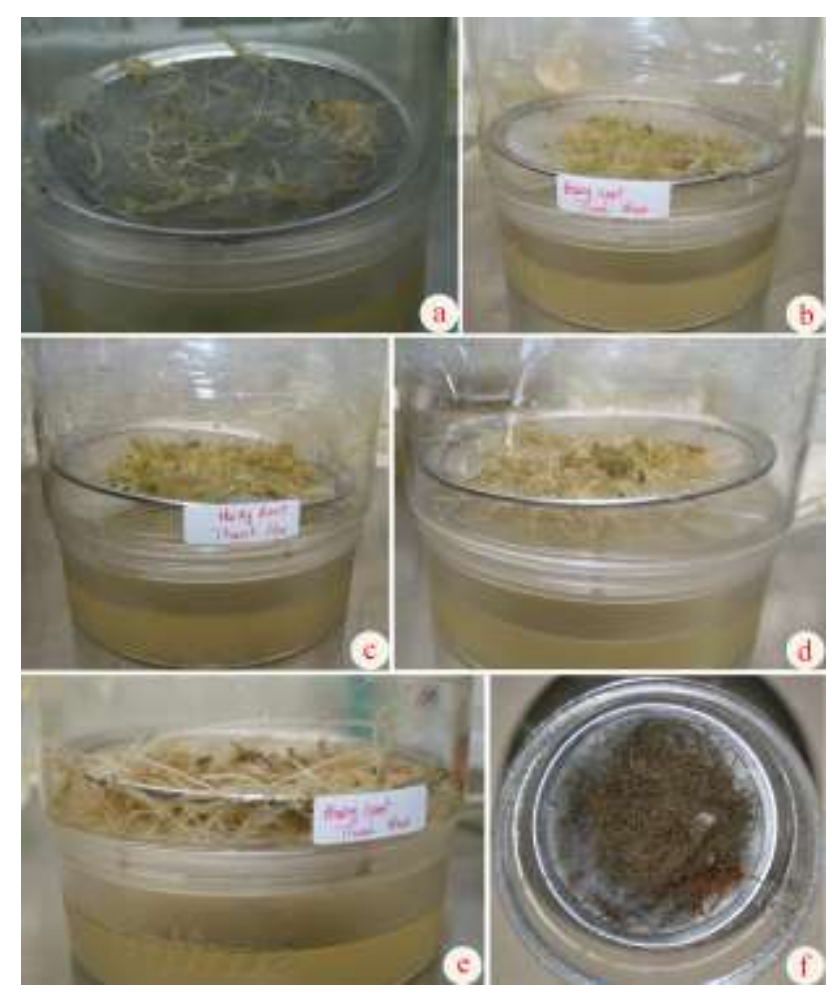

Figure 2. Dynamic of root growth in temporary immersion bioreactor at the dates after cultivation 5 (a), 10 (b), 15 (c), 20 (d) 25 (e) days and f was control

\section{Conclusion}

The B5 medium was suitable for root growth on semi-solid media. Strong root regeneration with culture media was found in leaves and supplemented with NAA $(0.5 \mathrm{mg} / \mathrm{l})$, BA, kinetin and IAA, IBA were not suitable for root growth. In vitro young leave were used as a transgenic plant material. The agrobacterium method proved to be effective. Agrobacteriummediated gene transfer was obtained. The hairy-root forms strongly on semi-solid B5 + NAA medium $(0.5 \mathrm{mg} / \mathrm{l})$ and was used as a raw material for liquid culture studies. The liquid medium MS was suitable for growing hairy-roots. The modified liquid MS medium corrects $\mathrm{NH}^{+} / \mathrm{NO}^{-}(5 / 25)$, sucrose $50 \mathrm{~g} / \mathrm{l}$, NAA supplementation $(0.5 \mathrm{mg} / \mathrm{l})$, improving artemisinin accumulation $1.468 \%$ compare to MS medium (no growth regulator) artemisinin accumulation $1.146 \%$.

\section{References}

[1] J. C. Laughlin, "Agricultural production of artemisinin--a review," PubMed (PMID: 8053017), 2001.

[2] N. B. Paniego and A. M. Giulietti, "Artemisinin production by Artemisia annua L.-transformed organ cultures," Enzyme Microb. Technol., vol. 18, pp. 526-530, 1996.

[3] A. Giri and L. Narasu, "Transgenic hairy roots: Recent trends and applications," Biotechnol. Adv., vol. 18, pp. 1-22, 2000.

[4] J. L. Han, H. Wang, H. C. Ye, Y. Liu, Z. Q. Li, Y. Zhang, Y. S. Zhang, F. Yan and G. F. Li, "High efficiency of genetic transformation and regeneration of Artemisia annua L. via Agrobacterium tumefaciensmediated procedure," Plant Science, vol. 168, pp. 73-80, 2005.

[5] D. Ramakrishnan and W. R. Curtis, "Fluid dynamic studies on plant root cultures for application to bioreactor design" in S. Furusaki and D. D. Ryu, Eds. Studies in plant science. Vol.4. Advances in plant biotechnology, Elsevier, 1994, pp281-305.

[6] P. Wilson, "The pilot-scale cultivation of transformed roots," in P. Doran, Eds. Hairy roots: Culture and applications. Amsterdam: Hardwood Academy Publishers, 1997, pp. 179-190.

[7] P. Weathers, T. Smith, D. Hemmavanh, E. Follansbee, J. Ryan and R. Cheetham, "Production of the antimalarial, artemisinin, by transformed roots of Artemisia annua," Acta Hort, vol. 426, pp. 157-163, 1996.

[8] M. Jaziri, K. Shimomura, K. Yoshimatsu, M. Fauconnier, M. Marlier and J. Homes, "Establishment of normal and transformed root cultures of Artemisia annua L. for artemisinin production," J Plant Physiol, vol. 145, pp. 175-177, 1995.

[9] C. Z. Liu, Y. C. Wang, X. Z. Kang and F. Ouyang, "Investigation of light, temperature and cultivated modes on growth and artemisinin synthesis of Artemisia annua L shoots," Acta Phytophy. Sin. Vol. 25, pp. 105-109, 1999.

[10] T. C. Smith, P. J. Weathers and R. D. Cheetham, "Effects of gibberellic acid on hairy root cultures of Artemisia annua: growth and artemisinin production," In Vitro Cell. Dev. Biol. Plant, vol. 33, pp. 75-79, 1997.

[11] P. J. Weathers, G. Bunk and M. McCoy, "The effect of phytohormones on growth and artemisinin production in Artemisia annua hairy roots," In Vitro Cell Dev. Biol. Plant, vol. 41, pp. 47-53, 2005.

[12] P. J. Weathers, R. D. Cheetham, E. Follansbee and T. Teoh, "Artemisinin production by transformed roots of Artemisia annua," Biotechnol. Lett, vol. 16, pp. 1281-1286, 1994.

[13] G. Q. Cai, G. Z. Li, H. C. Ye and G. F. Li, "Hairy root culture of Artemisia annua L. by Ri plasmid transformation and biosynthesis of artemisinin," Chin. J. Biotechnol, vol. 11, pp. 315-320, 1995.

[14] O. L. Gamborg, R. A. Miller and K. Ojima "Nutrient requirements of suspension cultures of soybean root cells," Exp. Cell Res, vol. 50, pp. $151-158,1968$ 
Proc. of The Seventh Intl. Conf. On Advances in Applied Science and Environmental Technology - ASET 2017 Copyright $(\odot$ Institute of Research Engineers and Doctors, USA .All rights reserved.

ISBN: 978-1-63248-136-8 doi: 10.15224/ 978-1-63248-136-8-26

[15] T. Murashige and F. Skoog, "A revised medium for rapid growth and bioassays with tobacco cultures," Physiol. Plant, vol. 15, pp. 473-497, 1962.

[16] J. D. Litvay, D. C. Verma and M. A. Johnson, "Influence of a loblolly pine (Pinus taeda L.) culture medium and its components on growth and somatic embryogenesis of the wild carrot (Daucus carota L.), " Plant Cell Report, vol. 4, pp. 325-328, 1985.

About Author (s):

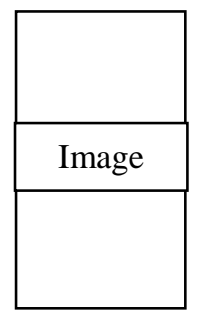

[Type a quote from the document or the summary of an interesting point. You can position the text box anywhere in the document. Use the Drawing Tools tab to change the formatting of the pull quote text box.] 